“ (C) 2017 IEEE. Personal use of this material is permitted. Permission from IEEE must be obtained for all other uses, in any current or future media, including

reprinting/republishing this material for advertising or promotional purposes, creating new collective works, for resale or redistribution to servers or lists, or reuse of any copyrighted component of this work in other works." 


\title{
An Object Oriented Bayesian Network Approach for Unsafe Driving Maneuvers Prevention System
}

\author{
Rinta Kridalukmana*, Hai Yan Lu*, Mohsen Naderpour* \\ *Decision Systems and e-Service Intelligence Laboratory \\ Centre for Artificial Intelligence \\ Faculty of Engineering and IT, University of Technology Sydney \\ rinta.kridalukmana@student.uts.edu.au \\ \{haiyan.lu,mohsen.naderpour\}@uts.edu.au
}

\begin{abstract}
As the main contributor to the traffic accidents, unsafe driving maneuvers have taken attentions from automobile industries. Although driving feedback systems have been developed in effort of dangerous driving reduction, it lacks of drivers awareness development. Therefore, those systems are not preventive in nature. To cover this weakness, this paper presents an approach to develop drivers awareness to prevent dangerous driving maneuvers. The approach uses ObjectOriented Bayesian Network to model hazardous situations. The result of the model can truthfully reflect a driving environment based upon situation analysis, data generated from sensors, and maneuvers detectors. In addition, it also alerts drivers when a driving situation that has high probability to cause unsafe maneuver to be detected. This model then is used to design a system, which can raise drivers awareness and prevent unsafe driving maneuvers.
\end{abstract}

Keywords-component; formatting; style; styling;

\section{INTRODUCTION}

Nowadays, the utilization of Driving Monitoring System (DMS) helps transportation and logistics industry to reduce operational cost and route efficiency by evaluating vehicles location history tracking. Moreover, the system also offers a number of safety-related features by detecting an over speed driving as one which produced by Teletrac Navman [1]. In addition to DMS, many researchers proposed to attach unsafe driving maneuvers detection to Advanced Driving Assistance Systems (ADAS) to contribute in accident risk reduction on the road. According to Wouters and Bos [2], notifying the drivers about their driving behavior could result in around 20\% reduction of accident risk. The New York State Police specifically defined that dangerous driving behaviors also include but not limited to: excessive speed, frequent or unsafe lane changes, and distracted driver by electronic device used during driving [3].

Although driving feedback is important to evaluate drivers so that they can change their driving habit in the future, it is good to enhance the drivers situation awareness before dangerous maneuvers occurred. For example, if a distance sensor system senses the signal that road is clear, an ADAS can warn drivers to keep driving in safe speed. Moreover, Krems, and Baumann [4] also noted that the drivers who get a notification for an upcoming event have a better response compared to those who are not warned.

So far, to minimize unsafe driving maneuvers, existing researches include (1) detecting unsafe driving events based on driving pattern, such as unsafe acceleration and lane change [5], swerving and weaving [6]; (2) predicting unsafe maneuvers based on drivers body movement and intent which observes drivers head, hand, and eye gaze [7-9]; (3) predicting unsafe driving maneuvers using probabilistic approaches from driving history data that can be used to estimate stop behavior at intersection [10] and unsafe lane change that will be made by drivers [11]. However, in helping drivers to make a good driving maneuvers decision, delivering situational information is very important. For example, drivers will make safer overtake decision if they know that the road speed limit will not be violated during overtaking. Moreover, the situational information also helps drivers to increase their Situation Awareness (SA) and to understand driving situation [12]. In the existing approach, this point have not been covered yet because they merely rely on finding driving maneuvers violation and learn when the violation will occur based on a particular characteristic.

This paper aims to apply the SA concept in a complex and dynamic driving situation to help in enhancing driver awareness purposely to avoid the unsafe driving maneuvers. Hence, this paper proposed a system that takes advantage from Object-Oriented Bayesian Network (OOBN) approach, which comprises dangerous maneuvers recognizer and situation analysis, a situational network based on OOBN to develop a hazardous situation model, and a notification system. With these features, the proposed system will be able to (1) deliver more effective way notify drivers in real time driving task to minimize unsafe driving maneuvers; (2) deliver more comprehensive information involving situational information to support drivers in making safe maneuvers decision; (3) improve drivers situation awareness.

The rest of the paper is organized in six sections. In Section II, background and related works are presented. The proposed system will be discussed in Section III. In Section IV and Section V, the system development approach and the way to evaluate the proposed system will be explained 


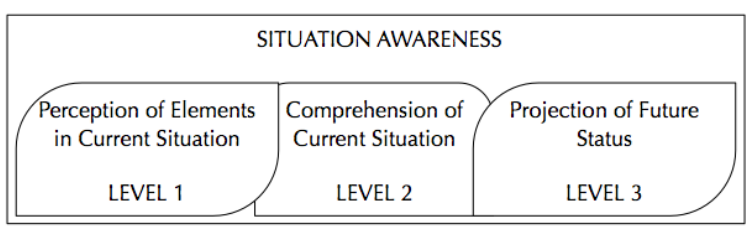

Figure 1. Situation Awareness Level by Endsley (1995)

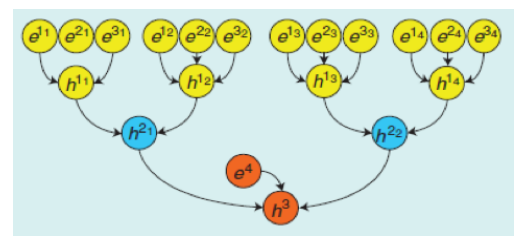

Figure 2. Object Oriented Bayesian Network[11] respectively. Finally, the conclusion will be drawn in Section VI.

\section{BACKGROUND AND RELATED WORKS}

\section{A. Situation Awareness}

Johannsdottir and Herdman [13] described SA as people interpretation and understanding about surrounding situation. Moreover, Endsley [14] defined individual awareness as a persons perception about situation or elements in a specific time and space to understand its meaning and make a future forecasting. Each person has a different ability to obtain SA for having different education, experience, and skills which may have an impact on their information processing. This background, then, contributes to form the basis of their SA during perception process in responding information displayed by the system or direct sensing. However, not all systems can provide information that easily to be understood by people or the system does not provide the adequate information about an ongoing hazardous situation. For example, an indicator lamp in a vehicle dashboard is turning on but there is no explanation or icon representation that refers to what kind of hazardous situation is occurring. Therefore, the SA involvement in system designing process should be considered to be able to deliver the better ongoing situation information to support the decision-making.

Furthermore, Endsley [7] viewed SA as the hierarchical phases which comprise three level of awareness (see Fig. 1). The first level is an interpretation of environments elements which refers to the needs of individual knowledge about their surrounding and their own conditions. For example, besides getting informed about their own vehicle, drivers should know other vehicles or barriers on the road and its dynamic status. The elements information, then, will develop the understanding of the current environment status which is defined as the second level of SA. In the third level, the individual understanding about their environment contributes to an ability to make a projection for the future actions. An instance case for levels two and three of SA is when drivers know that their own vehicle is in a safe distance with others, they can make a decision to make an overtaking maneuver.

\section{B. Dynamic and Object-Oriented Bayesian Network}

A Bayesian Network (BN) is a mathematical method that provides an ability to develop a model for a causal process containing uncertainty. This method uses graphical representation comprising a number of nodes and its possible relations among connected nodes. While classic BN tends to be static, Dynamic Bayesian Network (DBN) enables us to define several different variables at different time period. The definition of DBN is a pair $\left(B_{1}, 2 T B N\right)$ in which $B_{1}$ is Bayesian Network (BN) that defines a previous distribution $P\left(X_{t}\right)$ and $2 T B N$ is a two-slice temporal BN [15] with:

$$
P\left(X_{t} \mid X_{t-1}\right)=\prod_{i=1}^{n} P\left(X_{t}^{i} \mid P a\left(X_{t}^{i}\right)\right)
$$

where $X_{t}^{i}$ is a node at time frame $t$ and $P a\left(X_{t}^{i}\right)$ is the set of parent nodes that may exist in $t$ or $t-1$. At the first period of $2 T B N$, no parameter fills the nodes, but, each node will then be related to Conditional Probability Tables (CPT) or Conditional Probability Distribution (CPD). CPT commonly is used for discrete variables while CPD for continuous variables which, then, for all $\left.t>1, P\left(X_{t}^{i}\right)-P a\left(X_{t}^{i}\right)\right)$. The relations among periods of time represent the causal flow. The node $X_{t}^{i}$ is persistent when there is a relation from $X_{(t-1)}^{i}$ to $X_{t}^{i}$. Moreover, a relation during period of time is arbitrary, and directed relations reflect instantaneous causation. The DBN semantic can be defined by unrolling the $2 T B N$ until T time-slices exist. As join distribution result, Murphy [16] defined:

$$
P\left(X_{1: T}\right)=\prod_{t=1}^{T} \prod_{i=1}^{n} P\left(X_{t}^{i} \mid \operatorname{Pa}\left(X_{t}^{i}\right)\right)
$$

While DBN focuses on variables and its relationship, Object-oriented Bayesian Network (OOBN) provides a modeling approach by describing an inter-related object which can be in the physical or abstract form [17]. The object in this case is an abstraction of network fragment, which also called as instance node. For example (see Fig. 2), $h^{11}$ is an instance node that comprises variables $e^{11}, e^{21}$, and $e^{31}$ to describe $h^{11}$ [18]. Hence, this approach is also well-known as a hierarchical model. Kasper [18] demonstrated the ability of OOBN to recognize lane change maneuvers situation. Although this approach is similar with DBN, since there are levels of abstraction of network fragments, it is easier to review the process in OOBN.

\section{Proposed System}

The main goal of the proposed system is to develop driver awareness by delivering notification to drivers in 
critical situations that may trigger unsafe driving maneuvers. Moreover, it also generates driving feedback to make drivers able to change their behavior on the road. The schema of the proposed system can be seen in Fig. 3 .

\section{A. Sensor Reading}

There are numerous sensors usually used to detect vehicle maneuvers information such as in the vehicle Control Area Network (CAN), Global Positioning System (GPS) sensor, accelerometer, and distance sensor system. CAN is a direct sensing to the vehicle that contains information such as speed, acceleration, Revolutions Per Minute (RPM), and steer wheel angle. Meanwhile, the GPS sensors have geolocation coordinate information that comprises latitude, longitude, and timestamp. From this information, speed and acceleration can be inferred as well. Recently, some researchers also utilize accelerometer to detect driving maneuvers. Accelerometer data represents the movement of objects in three-axis system. While $\mathrm{x}$-axis and $\mathrm{y}$-axis represent lateral and longitudinal acceleration respectively, $\mathrm{z}$-axis represents normal acceleration.

\section{B. Unsafe Maneuvers Detector and Driving Situation Anal- ysis}

Basically, this component receives data from data acquisition tools which carry information either from vehicle, driver maneuvers, or from surrounding driving environment. To generate unsafe maneuvers information, the approach depends upon used sensing tools. The adoption of CAN in modern vehicles has changed the way of study to recognize the dangerous driving events, especially on how to collect information about vehicles operation. Data acquired using the CAN is typically more accurate than that of mobile sensors as it is directly connected to the vehicle [19]. Moreover, there are some additional adapters can be used for data acquisition through CAN interface, namely OBD-2 (On Board Diagnostics 2) and VBOX. Based on information generated from $\mathrm{CAN}$, researchers are able to identify several critical driving events, such as turn, lane change, stop and neutral driving [20, 21]. Also, Gonzalez et al. [22] determined aggressive drivers based on speed and acceleration information.

Although CAN is able to give accurate vehicles operation data, there are other alternative sensing technologies that can be utilized without having any direct connection to the vehicle. One of many popular devices in this category is GPS sensor that enables us to collect vehicles position periodically which includes latitude, longitude, and timestamp data. By calculating the trajectory data generated by GPS, the pattern of vehicles moving, such as over speed, abrupt acceleration, and direction [23], can be inferred. Additionally, other sensing tools such as accelerometer, gyroscope, and magnetometer are frequently used as well to recognize vehicles operation pattern. Interestingly, in

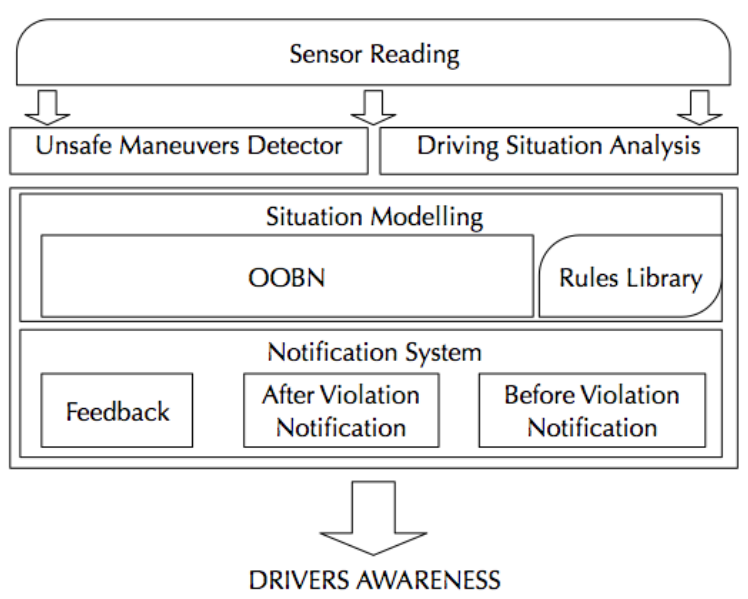

Figure 3. Proposed System

the latest smartphone technology, the functionality of GPS, accelerometer, gyroscope, and magnetometer are bundled on the smartphone device. The use of smartphones accelerometer was explored by Chen et al. [6] to identify a number of driving patterns including swerving, zigzag, swaying, sideslipping, fast turn, and fast U-turn respectively. However, since indirect sensing is used, there is a tradeoff between accuracy and information enrichment of vehicles operation.

As the internet and cloud technology reach the critical point in which the data connection cost is lower while the speed increases, many ideas have emerged to make the information of unsafe driving more useful, mainly for the industries. For instance, in insurance and transportation enterprises, this kind of information can be used for calculating risk of traffic accidents. Furthermore, early warning system can be developed to notify drivers regarding their bad habit on the road. Saiprasert [24] and G. Castignani et al. [25] have showed on their research how to send the driving information sensed by sensors to the remote server through internet for analysis and notify purposes and send the feedback to interested user. Other road and traffic information provided by third parties on cloud, such as Google Map and Open Map, may improve the identification of safe and unsafe driving since the limit speed can be different for each road. In addition, some of traffic information providers also offer web service enabling the different information among roads to be accessed easily. The internet ability to share traffic information may also be useful to support driving situation analysis. For example, drivers can have information about traffic jam and road construction work around them. Although it needs so much effort to deliver that kind of information, but in the future, it is possible. So far, to sense surrounding information, some vehicles use distance sensor technology. With this sensor, safe distance between vehicle and other objects on the road can be calculated. 


\section{Situation Modelling}

In this part, OOBN is developed to model driving situations which are represented by signals from user maneuvers detector and driving situation analysis component. Basically, a situation consists of related objects that have connection to the environment as well. Hence, in this case, every signal generated from the previous part of the system can be described as nodes in OOBN. Hence, each node can have several possible relations with other nodes that may lead to different level of hazardous situation information. For example, the combination of unsafe turning signal and crowded road signal will result in a higher risk than only unsafe turning signal occurred. For this purpose, a rules library covers each relationship among nodes and sets the level of risk of the hazardous situation.

In addition to determine the risk level of hazardous situation, the rules library also comprises a set of relationship information that might be used to recognize situations with high possibility when drivers do unsafe driving maneuvers. This function mainly relies on sensing surrounding environment ability with the support of sensors that can detect objects near the vehicle, or through shared traffic information provided by third party. Hence, driving situation analysis will have a significant influence on this module.

Each driver usually has several different characteristics during driving on the road. This driving profile can be reflected from the appearance probability of a particular set of situations. However, to achieve this purpose, information should be collected over some period of times as it needs to be validated at every point of time in the future. Once a particular characteristic is confirmed, system can generate several recommendations for future driving activities. Therefore, this feature can be considered to represent the learning ability of this component.

Although some hazardous situation decisions are based on observation in some period of time, there are several high-risk events that need to be informed to the driver immediately as a warning caused by their driving behavior. However, it needs an approach to send this notification because it might disturb drivers focus and lead accidents. To avoid this, the situation-aware friendly notification should be developed.

\section{Notification System}

The proposed system divides the notifications into three categories: feedback, before violation, and after violation notifications. In the feedback part, system provides information to drivers related to the type and number of violations occurred during a driving session. For example, information about how many times a driver creates unsafe turning or excessing the speed limit. Moreover, the feedback can give driving recommendation based on the characteristics learned from situation probabilistic in OOBN part.
Furthermore, there are some notifications that will be sent before violation occurred. In this case, violation refers to unsafe driving maneuvers. Basically, it will handle signals from OOBN and rules library components which send messages related to the situations that may have high probability for drivers to do a risky driving. The main purpose of delivering notification in the situation above is to prevent drivers that are trying to make unsafe driving events. For example, when the distance sensor system reads that the vehicle is not in safe distance with other objects and at the same time the acceleration tends to increase, the drivers will be warned to avoid the aggressive maneuvers. However, if drivers still make the dangerous movement, it will be handled by after violation notification part.

The drivers notification is a critical component that needs to be highlighted because it will relate to driver cognitive aspect during SA development. In addition, it becomes the part of the system that will be seen directly by drivers. Johannsdottir and Herdman [6] to understand a relation between working memory and SA discussed how working memory assists driver awareness in a hazardous situation in which sometimes in-cabin conversation with passengers cannot be avoided. Besides impairing driving performance, the drivers secondary task can degrade SA as well [26, 27]. Meanwhile, to integrate new slice information from surrounding will take time in the working memory. Therefore, it can be processed and at the same time can maintain drivers SA [4]. According to Lu, et al. [28], drivers need a few seconds to assess the underlying topology of driving situation. Yet, the time to be aware related to speed estimation is about 12-20 seconds. Although Soliman and Mathna [29] noted that the cognitive aspect of SA in the driving situation can be trained, Johannsdottir and Herdman [13] suggested that new way of informational technologies application should be developed by considering the role of visuospatial and phonological factors in cognitive aspect. In other words, the system like ADAS ideally can deliver information by compromising with the driver working memory.

\section{System DeVelopment}

The prototype development phase in this study uses Dynamic System Development technique (see Fig. 4). This technique comprises four activities: requirements, functional model, design and development, and implementation. In the stage of requirements, driving situation awareness aspects will be identified and transformed into technical specification. Subsequently, the functional model of the prototype will be set to direct the design and development stage. Finally, in the implementation, prototype will be tested and evaluated. Some problems that may emerge during this stage can be referred to previous stage. 


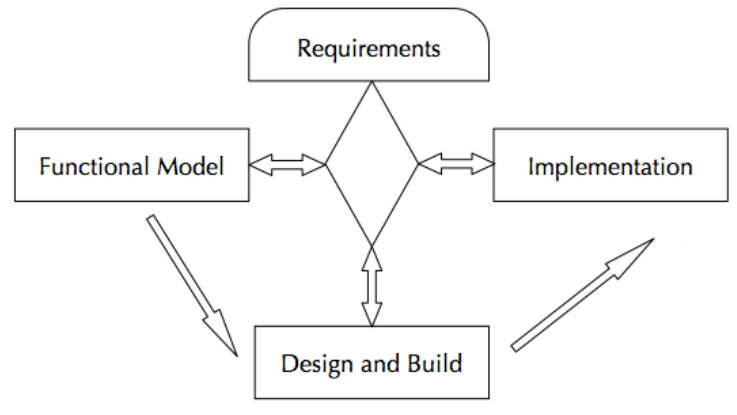

Figure 4. Dynamic System Development

\section{System Evaluation}

In this study, system evaluation will be highlighted to measure SA and OOBN performance. For SA measurement, Situation Awareness Global Assessment Technique (SAGAT) from Endsley [30] will be used to investigate the differences of SA among drivers and to assess the decisionmaking process through input reading. Meanwhile, to assess OOBN performance, sensitivity analysis will be conducted in accordance to three conditions: the probability changes comparison in parent nodes and child nodes, distribution of probability in parent nodes that have a consistent influence magnitude in child nodes, and total influence magnitude value from $\mathrm{x}$ attributes that should be always greater than its sub-attributes [31]

\section{CONCLUSION}

This study aims to develop drivers awareness by focusing on driver maneuvers and related driving environment. Hence, a new model to detect hazardous situation based on OOBN and to avoid unsafe driving maneuvers is proposed. The proposed system comprises three major components: unsafe driving maneuvers detector and driving situation analysis, situation modelling, and the notification system. For this purpose, the Human Computer Interface (HCI) is carefully designed to deliver driving feedback, before and after violation warning.

The contribution of this paper is to use OOBN that will deliver the probability of hazardous situation that can be used to learn the drivers characteristics and give a proper recommendation related to their driving behavior. By combining with the driving feedback feature that informs violation type and number to driver during a particular driving session, it will give an evaluation to driver. Thus, they can change their habit in the future.

For another contribution, the rule library also has a central role that comprises set relation among signals that reflect the existence of hazardous situations. This component will read some signals from detector and analyser part, and help the system to determine the priority level of critical situation.
Our future work will be divided into four phases: detector and analyser development, hazardous situation modelling and library development, notification system development, and evaluation of the overall system.

\section{ACKNOWLEDGMENT}

This work is supported by Indonesia Endowment Fund for Education (LPDP).

\section{REFERENCES}

[1] Teletrac Navman. (2017, 21 March). GPS Fleet Management Solution. Available: http://www.teletracnavman.com/

[2] P. I. J. Wouters and J. M. J. Bos, "Traffic accident reduction by monitoring driver behaviour with in-car data recorders," Accident Analysis \& Prevention, vol. 32, no. 5, pp. 643-650, 2000.

[3] New York State Governor's Traffic Safety Committee. (2017, 21 March). Aggresive Driving. Available: http://www.safeny.ny.gov/aggr-ndx.htm

[4] J. F. Krems and M. R. K. Baumann, "Driving and Situation Awareness: A Cognitive Model of Memory-Update Processes," in Human Centered Design: First International Conference, HCD 2009, Held as Part of HCI International 2009, San Diego, CA, USA, July 19-24, 2009 Proceedings, M. Kurosu, Ed. Berlin, Heidelberg: Springer Berlin Heidelberg, 2009, pp. 986994.

[5] M. Fazeen, B. Gozick, R. Dantu, M. Bhukhiya, and M. C. Gonzlez, "Safe driving using mobile phones," IEEE Transactions on Intelligent Transportation Systems, vol. 13, no. 3, pp. 1462-1468, 2012.

[6] Z. Chen, J. Yu, Y. Zhu, Y. Chen, and M. Li, "D 3: Abnormal driving behaviors detection and identification using smartphone sensors," in Sensing, Communication, and Networking (SECON), 2015 12th Annual IEEE International Conference on, 2015, pp. 524-532: IEEE.

[7] A. Doshi and M. M. Trivedi, "Tactical driver behavior prediction and intent inference: A review," in Intelligent Transportation Systems (ITSC), 2011 14th International IEEE Conference on, 2011, pp. 1892-1897: IEEE.

[8] E. Ohn-Bar, A. Tawari, S. Martin, and M. M. Trivedi, "Predicting driver maneuvers by learning holistic features," in Intelligent Vehicles Symposium Proceedings, 2014 IEEE, 2014, pp. 719-724: IEEE.

[9] A. Jain, H. S. Koppula, B. Raghavan, S. Soh, and A. Saxena, "Car that knows before you do: Anticipating maneuvers via learning temporal driving models," in 2015 IEEE International Conference on Computer Vision (ICCV), 2015, pp. 3182-3190.

[10] T. Kumagai and M. Akamatsu, "Prediction of human driving behavior using dynamic Bayesian networks," IEICE TRANSACTIONS on Information and Systems, vol. 89, no. 2, pp. 857-860, 2006.

[11] J. Firl and Q. Tran, "Probabilistic maneuver prediction in traffic scenarios," in ECMR, 2011, pp. 89-94.

[12] G. Underwood, A. Ngai, and J. Underwood, "Driving experience and situation awareness in hazard detection," Safety Science, vol. 56, pp. 29-35, 2013.

[13] K. R. Johannsdottir and C. M. Herdman, "The role of working memory in supporting drivers' situation awareness for surrounding traffic," Human Factors, vol. 52, no. 6, pp. 663673, 2010.

[14] M. R. Endsley, "Toward a theory of situation awareness in dynamic systems," Human Factors, vol. 37, no. 1, pp. 32-64, 1995. 
[15] M. Naderpour, J. Lu, and G. Zhang, "A fuzzy dynamic bayesian network-based situation assessment approach," in Fuzzy Systems (FUZZ), 2013 IEEE International Conference on, 2013, pp. 1-8: IEEE.

[16] K. P. Murphy, "Dynamic bayesian networks: representation, inference and learning," University of California, Berkeley, 2002.

[17] D. Koller and A. Pfeffer, "Object-oriented Bayesian networks," presented at the Proceedings of the Thirteenth conference on Uncertainty in artificial intelligence, Providence, Rhode Island, 1997.

[18] D. Kasper et al., "Object-oriented Bayesian networks for detection of lane change maneuvers," IEEE Intelligent Transportation Systems Magazine, vol. 4, no. 3, pp. 19-31, 2012.

[19] N. AbuAli and H. Abou-zeid, "Driver behavior modeling: Developments and future directions," International Journal of Vehicular Technology, vol. 2016, 2016.

[20] S. Choi, J. Kim, D. Kwak, P. Angkititrakul, and J. H. Hansen, "Analysis and classification of driver behavior using in-vehicle can-bus information," in Biennial Workshop on DSP for InVehicle and Mobile Systems, 2007, pp. 17-19.

[21] A. Sathyanarayana, P. Boyraz, and J. H. L. Hansen, ”Driver behavior analysis and route recognition by Hidden Markov Models," in 2008 IEEE International Conference on Vehicular Electronics and Safety, 2008, pp. 276-281.

[22] A. B. R. Gonzlez, M. R. Wilby, J. J. V. Daz, and C. S. vila, "Modeling and detecting aggressiveness from driving signals," IEEE Transactions on Intelligent Transportation Systems, vol. 15, no. 4, pp. 1419-1428, 2014.

[23] E. M. Carboni and V. Bogorny, "Inferring drivers behavior through trajectory analysis," in Intelligent Systems' 2014: Springer, 2015, pp. 837-848.

[24] C. Saiprasert and W. Pattara-Atikom, "Smartphone enabled dangerous driving report system," in 2013 46th Hawaii International Conference on System Sciences, 2013, pp. 1231-1237.

[25] G. Castignani, R. Frank, and T. Engel, "An evaluation study of driver profiling fuzzy algorithms using smartphones," in 2013 21st IEEE International Conference on Network Protocols (ICNP), 2013, pp. 1-6.

[26] A. Heenan, C. M. Herdman, M. S. Brown, and N. Robert, "Effects of conversation on situation awareness and working memory in simulated driving," Human Factors, vol. 56, no. 6, pp. 1077-1092, 2014.

[27] N. Schmig and B. Metz, "Three levels of situation awareness in driving with secondary tasks," Safety Science, vol. 56, pp. 44-51, 7// 2013.

[28] Z. Lu, X. Coster, and J. de Winter, "How much time do drivers need to obtain situation awareness? A laboratory-based study of automated driving," Applied Ergonomics, Article vol. 60, pp. 293-304, 2017.

[29] A. M. Soliman and E. K. Mathna, "Metacognitive strategy training improves driving situation awareness," Social Behavior \& Personality: an international journal, vol. 37, no. 9, pp. 11611170, 2009.

[30] M. R. Endsley, "Situation awareness global assessment technique (SAGAT)," in Aerospace and Electronics Conference, 1988. NAECON 1988., Proceedings of the IEEE $1988 \mathrm{Na}$ tional, 1988, pp. 789-795: IEEE.

[31] M. Naderpour and J. Lu, "A situation analysis decision support system based on dynamic object oriented Bayesian networks," Journal of Software, vol. 9, no. 8, pp. 2194-2199, 2014 